\title{
Midterm survival in patients treated for atrial fibrillation: A propensity-matched comparison to patients without a history of atrial fibrillation
}

Richard Lee, MD, MBA, Patrick M. McCarthy, MD, Edward C. Wang, PhD, Muthiah Vaduganathan, BA, Jane Kruse, RN, S. Chris Malaisrie, MD, and Edwin C. McGee, Jr, MD

Objective: Patients undergoing cardiac surgery with a history of untreated atrial fibrillation have reduced survival compared with similar patients without atrial fibrillation. We sought to compare the midterm survival of patients who received concomitant surgical ablation treatment for atrial fibrillation (atrial fibrillation ablated) with that of matched patients without a history of atrial fibrillation (no atrial fibrillation).

Methods: We evaluated 3262 consecutive patients (813 [25\%] with atrial fibrillation and 2449 [75\%] without preoperative atrial fibrillation) undergoing cardiac surgery at a single institution from April 2004 to April 2009. Of patients with atrial fibrillation, $565(70 \%)$ were treated with a concomitant surgical ablation procedure. Propensity scores were calculated on the basis of 37 known preoperative risk factors and yielded 744 patients. Midterm survival was compared between patients with atrial fibrillation ablation $(\mathrm{n}=372)$ and patients without atrial fibrillation $(\mathrm{n}=372)$. Survival was also compared between patients with successful vs unsuccessful ablation, and a matched analysis was performed at 1 year between the 2 groups.

Results: Mean follow-up was $2.7 \pm 1.6$ years. Patients without atrial fibrillation and patients with treated atrial fibrillation had similar early 30 -day mortality $(1.2 \%$ vs $0.3 \%, P=.37)$ and overall mortality rates $(11.6 \%$ vs $9.4 \%, P=.344)$, respectively. Survival analysis showed no differences at 1,3 , and 5 years between the 2 groups ( $\log$-rank $P=.22$ ). At last follow-up, $78 \%$ of treated patients were free of atrial fibrillation. At 1 year, $68 \%$ of patients were free of atrial fibrillation and antiarrhythmic medication. Freedom from atrial fibrillation and antiarrhythmic medication at 1 year predicted improved midterm survival $(P=.03)$ compared with patients in atrial fibrillation or taking antiarrhythmic medication. Propensity-matched analysis after 1 year demonstrated improved survival for patients who were successfully treated $(P=.016)$.

Conclusions: Patients undergoing surgical treatment of atrial fibrillation had survival similar to that of patients without a history of atrial fibrillation. Those with successful sinus restoration had improved survival compared with those who were treated but remained in atrial fibrillation. (J Thorac Cardiovasc Surg 2012;143:1341-51)

Earn CME credits at

http://cme.ctsnetjournals.org

Patients with atrial fibrillation $(\mathrm{AF})$ have a reduced survival when compared with patients in sinus rhythm. ${ }^{1}$ Patients with AF have increased rates of stroke, heart failure, and

\footnotetext{
From the Bluhm Cardiovascular Institute, Division of Cardiac Surgery at Northwestern University Feinberg School of Medicine and Northwestern Memorial Hospital, Chicago, Ill.

Disclosures: Authors have nothing to disclose with regard to commercial support.

Read at the 37th Annual Meeting of The Western Thoracic Surgical Association, Colorado Springs, Colorado, June 22-25, 2011.

Received for publication June 25, 2010; revisions received Jan 12, 2012; accepted for publication Feb 3, 2012; available ahead of print April 2, 2012.

Address for reprints: Richard Lee, MD, MBA, Division of Cardiac Surgery, Northwestern University, 201 East Huron Street, Suite 11-140, Chicago, IL 606112968 (E-mail: ricklee@nmh.org).

$0022-5223 / \$ 36.00$

Copyright (c) 2012 by The American Association for Thoracic Surgery doi:10.1016/j.jtcvs.2012.02.006
}

all-cause mortality. ${ }^{2}$ Pharmacologic attempts at sinus restoration have been unable to establish a survival advantage over rate control alone. ${ }^{3}$ However, on subsequent multivariate analysis, patients with successful restoration of sinus rhythm demonstrated a survival advantage over those in $\mathrm{AF}$, suggesting that a therapy that successfully treats $\mathrm{AF}$ may affect survival. ${ }^{4}$

Patients with preoperative $\mathrm{AF}$ undergoing cardiac surgery also have an increased risk of mortality. ${ }^{5-9}$ For example, patients with AF undergoing coronary artery bypass grafting (CABG) have a $24 \%$ survival disadvantage at 10 years compared with matched patients undergoing $\mathrm{CABG}$ without preoperative $\mathrm{AF}^{5,6}$ In matched patients undergoing aortic valve replacement, patients with AF have worse survival and an increased incidence of strokes and heart failure.,

Surgical ablation of AF in patients undergoing cardiac surgery has improved and is more widely applied, but it is still performed in the minority of cases. ${ }^{10}$ Ablation usually adds little to the operation ( 9 minutes to crossclamp time and 9 minutes to cardiopulmonary bypass time in patients 


$$
\begin{aligned}
& \text { Abbreviations and Acronyms } \\
& \begin{aligned}
\text { AA } & =\text { antiarrhythmic } \\
\text { AF } & =\text { atrial fibrillation } \\
\text { CABG } & =\text { coronary artery bypass grafting } \\
\text { CI } & =\text { confidence interval } \\
\text { HR } & =\text { hazard ratio }
\end{aligned}
\end{aligned}
$$

undergoing mitral valve surgery). ${ }^{10}$ Surgery for AF has been shown to be safe and effective and does not add to perioperative morbidity and mortality when combined with other cardiac procedures; however, a benefit to midterm survival has not been established. ${ }^{11-15}$

The purpose of this study was to compare the midterm survival of patients with $\mathrm{AF}$ who received surgical ablation for $\mathrm{AF}$ ( $\mathrm{AF}$ ablated) at the time of cardiac surgery with that of similar patients without preoperative AF (no AF). Our hypotheses were that (1) the AF ablated group would have a similar survival as the no AF group after adjusting for preoperative risk factors, and that (2) the successful restoration of sinus rhythm would improve survival compared with patients who return to AF.

\section{MATERIALS AND METHODS}

We queried the Bluhm Cardiovascular Institute's Clinical Trials Cardiac Surgery Outcomes Registry for all patients who underwent cardiac surgery since the inception of the database from April 2004 to April 2009. This Registry is approved by the institutional review board at Northwestern University (project STU00012288). Data were collected from patients enrolled in the Registry and from medical record review. All patients in this study consented for the use of their follow-up data. Data were deidentified before analysis.

\section{Study Population}

We evaluated 3262 consecutive patients (813 [25\%] with AF and 2449 [75\%] without preoperative AF). Of the 813 patients with AF, $565(70 \%)$ were treated with a surgical AF ablation procedure (classic cut-and-sew Maze in 78, biatrial Maze in 140, left atrial Maze in 213, pulmonary vein isolation [island] in 108 , pulmonary vein isolation [box] in 23 , and procedures that not fit into any other category in 3). The AF untreated $(n=248)$ group had similar age but several adverse preoperative risk factors, including diabetes, hypertension, congestive heart failure, New York Heart Association class, renal failure, chronic obstructive pulmonary disease, peripheral vascular disease, myocardial infarction, stroke, and transient ischemic attack compared with the AF ablated group (Table 1). Patients were treated for AF when the risks of adding the procedure were considered to be low, there was a reasonable chance for success, and the surgery was performed by an experienced surgeon in accordance with the Heart Rhythm Society/European Heart Rhythm Association/European Cardiac Arrhythmia Society Expert Consensus Statement. ${ }^{16}$ Preoperative demographic and clinical data were collected on all patients, as were perioperative outcomes. The standard Society of Thoracic Surgeons' database definitions were used for all variables. The Society of Thoracic Surgeons' risk score could not be implemented in those with AF; therefore, Ambler risk scores were calculated and compared. Midterm survival was identified by the Social Security Death Index and measured as time (days) to death or last follow-up from date of surgery. Follow-up was $100 \%$ complete.

\section{Statistical Analysis}

Before final statistical analysis, preliminary data were analyzed using univariate and graphic methods wherever applicable to facilitate inspection and interpretation of the data. Outliers and influential observations were identified and checked for accuracy. Data error due to data entry oversight was appropriately corrected. Data were summarized using descriptive statistics (eg, means and standard deviation for continuous variables; count and frequency for categoric variables). Group differences in patient demographics and clinical and surgical characteristics were compared using a $t$ test (parametric) or Wilcoxon Mann-Whitney test (nonparametric) for continuous variables and chi-square test or Fisher exact test for categoric data.

Unadjusted survival estimates were analyzed using the Kaplan-Meier method, and adjusted hazard ratio (HR) of long-term mortality was estimated using the Cox proportional hazard models. Risk factors by which these survival estimates were adjusted for are shown in Table 2. Propensity scores were further calculated on the basis of the probability of undergoing a surgical $\mathrm{AF}$ ablation procedure and no preoperative $\mathrm{AF}$ condition. The propensity score was developed by fitting a logistic regression model with $\mathrm{AF}$ treatment as the outcome variable and all risk factors in Table 1 as the predictor variables. Missing data of predictor variables were imputed using multiple imputations of SAS PROC MI (SAS Institute Inc, Cary, NC) procedure. All $P$ values after propensity score adjustment were not statistically significant (Table 2), indicating a good balance after adjustment.

The AF ablated group $(\mathrm{n}=372)$ was propensity matched (1:1) with the no AF history $(\mathrm{n}=372)$ group using the caliper-matching method. ${ }^{17}$ Specifically, we imposed a 0.02 propensity score tolerance on the maximum propensity score distance (caliper) in our matching algorithm so that bad matches could be avoided. Balance of covariates before and after propensity adjustment was checked using both density distribution of the propensity score and Wald chi-square statistics to access the quality of the match. The 744 matched patients were analyzed for differences in midterm survival using Kaplan-Meier and log-rank methodology. Additional matches were performed for patients undergoing isolated aortic valve replacement $(n=44)$, mitral valve repair or replacement $(n=130)$, and concomitant coronary artery bypass and valve intervention $(\mathrm{CABG}+$ valve $)(\mathrm{n}=186)$.

For the AF ablated group, the standard protocol for postoperative monitoring and medication management of AF at Bluhm Cardiovascular Institute, developed in collaboration with cardiac electrophysiologists, was reviewed with patients and shared with referring cardiologists. The protocol recommends continuous electrocardiographic monitoring for a minimum of 24 hours at 3 and 6 months to guide medication changes and every 6 months for 2 years after intervention to monitor rhythm status. Pacemaker interrogation was used whenever possible. If any monitor showed AF and no further attempts were made to restore sinus rhythm, the patient was deemed a "failure" and continuous monitoring was no longer required. Patients were also contacted by phone at 3,6, and 12 months to track medications and provide support.

Survival was compared in the AF ablation group between patients with successful sinus restoration and patients with "failed" treatment. Success was defined at 1 year as freedom from of any episode of AF without antiarrhythmic (AA) medications. Throughout the report, statistical significance was established at an alpha level of .05. All statistical analyses were performed using SAS 9.2 statistical software (SAS Institute Inc). In an effort to assess whether the return to AF was a marker or cause of mortality, after 1 year, 77 patients successfully treated were propensity matched (1:1) with 77 patients with unsuccessful treatment using the caliper matching method. ${ }^{17,18}$ We again imposed a 0.02 propensity score tolerance on the maximum propensity score distance (caliper) in our matching algorithm so that bad matches could be avoided. Covariate balance before and after propensity adjustment was checked using both density distribution of the propensity score and Wald chi-square statistics to access the quality of the match. The 154 matched patients were analyzed for differences in midterm survival using Kaplan-Meier and log-rank methodology. 
TABLE 1. Comparison of characteristics in the AF population: AF ablated versus AF untreated

\begin{tabular}{|c|c|c|c|c|c|}
\hline \multirow[b]{2}{*}{ Variables } & \multicolumn{2}{|c|}{ AF ablated $(N=565)$} & \multicolumn{2}{|c|}{ AF untreated $(N=248)$} & \multirow[b]{2}{*}{$P$ valuc } \\
\hline & $\mathbf{n}$ & $\%$ & $\mathbf{n}$ & $\%$ & \\
\hline \multicolumn{6}{|l|}{ Demographics } \\
\hline Age $($ mean $\pm \mathrm{SD})$ & \multicolumn{2}{|c|}{$65.9 \pm 12.2$} & \multicolumn{2}{|c|}{$66.5 \pm 15.1$} & .5628 \\
\hline Male & 329 & 58.2 & 173 & 69.8 & .0018 \\
\hline Race & & & & & .0376 \\
\hline White & 435 & 77.0 & 194 & 78.2 & \\
\hline AA & 26 & 4.6 & 20 & 8.1 & \\
\hline Hispanic & 10 & 1.8 & 7 & 2.8 & \\
\hline Asian & 11 & 2.0 & 5 & 2.0 & \\
\hline Other & 83 & 14.7 & 22 & 8.9 & \\
\hline \multicolumn{6}{|l|}{ Health characteristics } \\
\hline $\mathrm{CHADS}_{2}($ mean $\pm \mathrm{SD})$ & \multicolumn{2}{|c|}{$1.6 \pm 1.2$} & \multicolumn{2}{|c|}{$2.3 \pm 1.3$} & $<.0001$ \\
\hline $\mathrm{BMI}($ mean $\pm \mathrm{SD})$ & \multicolumn{2}{|c|}{$27.5 \pm 5.9$} & \multicolumn{2}{|c|}{$27.7 \pm 6.5$} & .7728 \\
\hline Smoker & 149 & 26.4 & 68 & 27.4 & .7559 \\
\hline Diabetes & 77 & 13.6 & 66 & 26.6 & $<.0001$ \\
\hline Hypertension & 321 & 56.8 & 164 & 66.1 & .0127 \\
\hline $\mathrm{CHF}$ & 206 & 36.5 & 149 & 60.1 & $<.0001$ \\
\hline NYHA & & & & & $<.0001$ \\
\hline Class 1 & 121 & 21.4 & 19 & 7.7 & \\
\hline Class 2 & 246 & 43.5 & 65 & 26.2 & \\
\hline Class 3 & 173 & 30.6 & 91 & 36.7 & \\
\hline Class 4 & 25 & 4.4 & 73 & 29.4 & \\
\hline Renal failure & 24 & 4.2 & 30 & 12.1 & $<.0001$ \\
\hline Previous dialysis & 5 & 0.9 & 11 & 4.4 & .0008 \\
\hline COPD & 71 & 12.6 & 58 & 23.4 & .0001 \\
\hline PVD & 26 & 4.6 & 29 & 11.7 & .0002 \\
\hline Moderate pulmonary HTN & 188 & 33.3 & 79 & 31.9 & .6915 \\
\hline Severe pulmonary HTN & 130 & 23.0 & 67 & 27.0 & .2195 \\
\hline MI & 58 & 10.3 & 69 & 27.8 & $<.0001$ \\
\hline Angina & 70 & 12.4 & 43 & 17.3 & .0603 \\
\hline Stroke/TIA & 57 & 10.1 & 46 & 18.5 & .0008 \\
\hline Family history CAD & 105 & 18.6 & 38 & 15.3 & .2607 \\
\hline \multicolumn{6}{|l|}{ Surgical characteristics } \\
\hline No. of diseased vessels (mean $\pm \mathrm{SD}$ ) & \multicolumn{2}{|c|}{$0.8 \pm 1.2$} & \multicolumn{2}{|c|}{$1.3 \pm 1.3$} & $<.0001$ \\
\hline Left main disease & 30 & 5.4 & 32 & 13.2 & .0001 \\
\hline Preoperative creatinine (mean \pm SD) & \multicolumn{2}{|c|}{$1.17 \pm 0.8$} & \multicolumn{2}{|c|}{$1.46 \pm 1.1$} & .0002 \\
\hline Surgical incidence & & & & & $<.0001$ \\
\hline 1 st & 465 & 82.3 & 137 & 55.2 & \\
\hline 2nd & 90 & 15.9 & 93 & 37.5 & \\
\hline $3+$ & 10 & 1.8 & 18 & 7.3 & \\
\hline Indication of surgery & & & & & $<.0001$ \\
\hline Elective & 511 & 90.4 & 138 & 55.7 & \\
\hline Emergency & 1 & 0.2 & 10 & 4.0 & \\
\hline Urgent & 53 & 9.4 & 100 & 40.3 & \\
\hline Type of surgery & & & & & \\
\hline CABG & 172 & 30.4 & 83 & 33.5 & .3920 \\
\hline Aortic valve & 167 & 29.6 & 107 & 43.1 & .0002 \\
\hline Mitral valve & 341 & 60.4 & 51 & 20.6 & $<.0001$ \\
\hline Tricuspid valve & 162 & 28.7 & 42 & 16.9 & .0004 \\
\hline Valve + CABG & 141 & 25.0 & 50 & 20.2 & .1376 \\
\hline Previous medications & & & & & \\
\hline ACE & 201 & 35.7 & 87 & 35.2 & .8957 \\
\hline $\mathrm{ADP}$ & 9 & 1.6 & 12 & 4.9 & .0071 \\
\hline Aspirin & 121 & 21.4 & 96 & 38.9 & $<.0001$ \\
\hline Beta-blockers & 362 & 64.1 & 138 & 55.6 & .0230 \\
\hline
\end{tabular}


TABLE 1. Continued

\begin{tabular}{|c|c|c|c|c|c|}
\hline \multirow[b]{2}{*}{ Variables } & \multicolumn{2}{|c|}{ AF ablated $(N=565)$} & \multicolumn{2}{|c|}{ AF untreated $(N=248)$} & \multirow[b]{2}{*}{$P$ value } \\
\hline & $\mathbf{n}$ & $\%$ & $\mathbf{n}$ & $\%$ & \\
\hline Warfarin & 88 & 15.6 & 69 & 28.0 & $<.0001$ \\
\hline Nonstatin & 28 & 5.0 & 17 & 6.9 & .2756 \\
\hline Statin & 240 & 42.5 & 120 & 48.4 & .1183 \\
\hline \multicolumn{6}{|l|}{ Discharge medications } \\
\hline $\mathrm{ACE}$ & 129 & 23.7 & 50 & 23.0 & .8535 \\
\hline $\mathrm{ADP}$ & 17 & 3.1 & 18 & 8.0 & .0026 \\
\hline Aspirin & 500 & 90.1 & 206 & 91.6 & .5269 \\
\hline Beta-blockers & 381 & 70.0 & 151 & 67.1 & .4241 \\
\hline Warfarin & 472 & 85.2 & 121 & 53.5 & $<.0001$ \\
\hline Nonstatin & 23 & 4.1 & 13 & 5.2 & .4548 \\
\hline Statin & 406 & 71.9 & 160 & 64.5 & .03613 \\
\hline Ambler score (mean $\pm \mathrm{SD}$ ) & \multicolumn{2}{|c|}{$8.4 \pm 8.2$} & \multicolumn{2}{|c|}{$12.5 \pm 9.8$} & $<.0001$ \\
\hline
\end{tabular}

$A F$, Atrial fibrillation; $S D$, standard deviation; $A A$, antiarrhythmic; $C H A D S_{2}$, Congestive heart failure, Hypertension, Age $\geq 75$ years, Diabetes, previous Stroke; $B M I$, body mass index; $C H F$, congestive heart failure; $N Y H A$, New York Heart Association; $C O P D$, chronic obstructive pulmonary disease; $P V D$, peripheral vascular disease; $H T N$, hypertension; $M I$, myocardial infarction; $T I A$, transient ischemic attack; $C A D$, coronary artery disease; $C A B G$, coronary artery bypass grafting; $A C E$, angiotensin-converting enzyme; $A D P$, adenosine diphosphate.

\section{RESULTS}

There was no difference in 30-day mortality $(0.3 \%$ vs $1.2 \%, P=.4$ ) (Ambler score predicted mortality $6.9 \%$ and $6.3 \%, P=.22)$, postoperative stroke $(2.4 \%$ vs $4.1 \%$, $P=.15$ ), length of stay ( 8 vs 9 days, $P=.3$ ), intensive care unit stay (77 vs 80 hours, $P=.8$ ), or length of follow-up (2.8 vs 2.7 years, $P=.12$ ) between the propensity-matched AF ablated and no AF groups, respectively.

In the unadjusted model, patients undergoing surgery with a history of AF had reduced survival compared with patients with no AF (Figure $1, P<.0001$ ). The unadjusted HR was 1.65 (95\% confidence interval [CI], 1.33-2.04). The AF ablated group had better survival than the AF untreated group (HR, 0.39; 95\% CI, 0.27-0.55) and similar survival to the no AF group (HR, 1.15; 95\% CI, 0.881.51) (Figure 2).

There was no difference in mortality between the matched $\mathrm{AF}$ ablated and no AF groups $(P=.22$, Figure 3). Both groups illustrated similar patient survival at 1,3 , and 5 years $(95 \%$ vs $94 \%, 90 \%$ vs $87 \%$, and $87 \%$ vs $82 \%$ for AF ablated vs no AF, respectively). Additional comparisons were performed between matched patients undergoing isolated aortic valve replacement, mitral valve replacement, and $\mathrm{CABG}+$ valve. There was no difference in survival between patients in the AF ablated group and patients in the no AF group undergoing any of these procedures.

Follow-up at 1 year was available for 336 patients in the AF ablated group. A total of 225 of these patients $(67 \%)$ had no episode of AF lasting more than 30 seconds within the first year and were free of AA medications and classified as having "successful treatment." Freedom from AF and AA medications at 1 year independently predicted long-term survival compared with patients in whom ablation failed (unadjusted $P=.0003$ and adjusted $P=.03$ ) (Figure 4). At last follow-up, $78 \%$ of patients were free of AF. There was a trend for improved survival in this group $(P=.06)$ compared with treated patients who remained in AF.

After 1 year, survivors who were successfully treated were similar in many ways to those patients whose treatment failed (Table 3). However, they were younger, had lower serum creatinine, and were more likely to have no prior cardiac operation. Seventy-seven patients who were successfully treated after 1 year were matched with 77 patients who had failed treatment (in AF or taking AA medication). Survival was different between these groups (Figure 5) and favored those patients who were successfully treated.

\section{DISCUSSION}

The published literature suggests that patients with AF undergoing cardiac surgery have reduced survival compared with patients in sinus rhythm..$^{5-9}$ Our article demonstrates that when select patients are treated for AF at the time of cardiac surgery, their midterm survival is the same as those without a history of AF. Further, successful restoration of sinus rhythm improves survival compared with treated patients who remain in AF, a finding analogous to the Atrial Fibrillation Follow-Up Investigation of Rhythm Management trial. ${ }^{4}$ This finding persists even with the passage of time and the change of the patient population. ${ }^{18}$

Patients undergoing cardiac surgery represent a unique population. Adding a concomitant AF procedure does not add much to the operation but results in sinus restoration approximately $80 \%$ of the time $e^{13,19-23}$ (in our study $78 \%$, including $67 \%$ free of $\mathrm{AA}$ medications and $\mathrm{AF}$ ). At least 6 randomized trials in patients undergoing mitral valve surgery have shown that treating $\mathrm{AF}$ is more likely to restore sinus rhythm than nontreatment. ${ }^{14,24-29}$ However, 
TABLE 2. Comparison of characteristics of AF ablated versus no AF populations: Total sample and propensity-matched groups

\begin{tabular}{|c|c|c|c|c|c|c|c|c|c|c|}
\hline \multirow[b]{3}{*}{ Variables } & \multicolumn{5}{|c|}{ Total sample $(\mathrm{N}=\mathbf{3 0 1 4})$} & \multicolumn{5}{|c|}{ Propensity-matched sample $(\mathrm{N}=744)$} \\
\hline & \multicolumn{2}{|c|}{ AF ablated $(N=565)$} & \multicolumn{2}{|c|}{ No AF $(N=2449)$} & \multirow[b]{2}{*}{$P$ value } & \multicolumn{2}{|c|}{ AF ablated $(N=372)$} & \multicolumn{2}{|c|}{ No AF $(\mathbf{N}=372)$} & \multirow[b]{2}{*}{$P$ value } \\
\hline & $\mathbf{n}$ & $\%$ & $\mathbf{n}$ & $\%$ & & $\mathbf{n}$ & $\%$ & $\mathbf{n}$ & $\%$ & \\
\hline \multicolumn{11}{|l|}{ Demographics } \\
\hline Age (mean \pm SD) & \multicolumn{2}{|c|}{$65.9 \pm 12.2$} & \multicolumn{2}{|c|}{$61.7 \pm 13.8$} & $<.0001$ & \multicolumn{2}{|c|}{$64.8 \pm 12.5$} & \multicolumn{2}{|c|}{$64.7 \pm 13.1$} & .9088 \\
\hline Male & 329 & 58.2 & 1644 & 67.1 & $<.0001$ & 224 & 60.2 & 231 & 62.1 & .5985 \\
\hline Race & & & & & $<.0001$ & & & & & .8932 \\
\hline White & 435 & 77.0 & 1718 & 70.2 & & 279 & 75.0 & 282 & 75.8 & \\
\hline AA & 26 & 4.6 & 235 & 9.6 & & 23 & 6.2 & 28 & 7.5 & \\
\hline Hispanic & 10 & 1.8 & 104 & 4.3 & & 7 & 1.9 & 6 & 1.6 & \\
\hline Asian & 11 & 2.0 & 63 & 2.6 & & 8 & 2.2 & 6 & 1.6 & \\
\hline Other & 83 & 14.7 & 329 & 13.4 & & 55 & 14.8 & 50 & 13.4 & \\
\hline \multicolumn{11}{|l|}{ Health characteristics } \\
\hline $\mathrm{CHADS}_{2}($ mean $\pm \mathrm{SD})$ & \multicolumn{2}{|c|}{$1.6 \pm 1.2$} & & & .0357 & & & & & .8519 \\
\hline $\mathrm{BMI}($ mean $\pm \mathrm{SD})$ & & & 28. & 5.8 & .0751 & & & & 6.0 & .4750 \\
\hline Smoker & 149 & 26.4 & 764 & 31.2 & .0245 & 95 & 25.5 & 114 & 30.7 & .1212 \\
\hline Diabetes & 77 & 13.6 & 515 & 21.0 & $<.0001$ & 56 & 15.1 & 61 & 16.4 & .6146 \\
\hline Hypertension & 321 & 56.8 & 1554 & 63.5 & .0033 & 223 & 60.0 & 223 & 60.0 & 1.0000 \\
\hline $\mathrm{CHF}$ & 206 & 36.5 & 580 & 23.7 & $<.0001$ & 120 & 32.3 & 129 & 34.7 & .4844 \\
\hline NYHA & & & & & $<.0001$ & & & & & .8771 \\
\hline Class 1 & 121 & 21.4 & 675 & 27.6 & & 89 & 23.9 & 84 & 22.6 & \\
\hline Class 2 & 246 & 43.5 & 928 & 37.9 & & 163 & 43.8 & 159 & 42.7 & \\
\hline Class 3 & 173 & 30.6 & 640 & 26.1 & & 105 & 28.2 & 115 & 30.9 & \\
\hline Class 4 & 25 & 4.4 & 205 & 8.4 & & 15 & 4.0 & 14 & 3.8 & \\
\hline Renal failure & 24 & 4.2 & 119 & 4.9 & .5366 & 11 & 3.0 & 14 & 3.8 & .5416 \\
\hline Previous dialysis & 5 & 0.9 & 61 & 2.5 & .0187 & 3 & 0.8 & 3 & 0.8 & 1.0000 \\
\hline COPD & 71 & 12.6 & 255 & 10.4 & .1373 & 44 & 11.8 & 49 & 13.2 & .5794 \\
\hline PVD & 26 & 4.6 & 213 & 8.7 & .0017 & 17 & 4.6 & 23 & 6.2 & .3294 \\
\hline Moderate pulmonary HTN & 188 & 33.3 & 491 & 20.0 & $<.0001$ & 112 & 30.1 & 115 & 30.9 & .8112 \\
\hline Severe pulmonary HTN & 130 & 23.0 & 251 & 10.2 & $<.0001$ & 74 & 19.9 & 75 & 20.2 & .9270 \\
\hline MI & 58 & 10.3 & 496 & 20.3 & $<.0001$ & 39 & 10.5 & 50 & 13.4 & .2140 \\
\hline Angina & 70 & 12.4 & 824 & 33.6 & $<.0001$ & 55 & 14.8 & 58 & 15.6 & .7593 \\
\hline Stroke/TIA & 57 & 10.1 & 206 & 8.4 & .2030 & 32 & 8.6 & 29 & 7.8 & .6885 \\
\hline Family history CAD & 105 & 18.6 & 513 & 20.9 & .2098 & 70 & 18.8 & 74 & 19.9 & .7105 \\
\hline Surgical characteristics & & & & & & & & & & \\
\hline No. of diseased vessels (mean $\pm \mathrm{SD}$ ) & & & & & $<.0001$ & & & & & .1065 \\
\hline Left main disease & 30 & 5.4 & 380 & 15.7 & $<.0001$ & 23 & 6.2 & 31 & 8.3 & .2583 \\
\hline Preoperative creatinine (mean \pm SD) & & & 1.1 & 0.9 & .5784 & & & & 0.5 & .9887 \\
\hline Surgical incidence & & & & & .0010 & & & & & .8314 \\
\hline 1 st & 465 & 82.3 & 2148 & 87.7 & & 309 & 83.1 & 303 & 81.5 & \\
\hline 2 nd & 90 & 15.9 & 255 & 10.4 & & 55 & 14.8 & 61 & 16.4 & \\
\hline $3+$ & 10 & 1.8 & 46 & 1.9 & & 8 & 2.2 & 8 & 2.2 & \\
\hline Indication of surgery & & & & & $<.0001$ & & & & & .9014 \\
\hline Elective & 511 & 90.4 & 1889 & 77.1 & & 335 & 90.1 & 337 & 90.6 & \\
\hline Emergency & 1 & 0.2 & 55 & 2.3 & & 0 & 0 & 0 & 0 & \\
\hline Urgent & 53 & 9.4 & 505 & 20.6 & & 37 & 9.9 & 36 & 9.4 & \\
\hline Type of surgery & & & & & & & & & & \\
\hline CABG & 172 & 30.4 & 1305 & 53.3 & $<.0001$ & 122 & 32.8 & 140 & 37.6 & .1671 \\
\hline Aortic valve & 167 & 29.6 & 891 & 36.4 & .0022 & 128 & 34.4 & 121 & 32.5 & .5865 \\
\hline Mitral valve & 341 & 60.4 & 590 & 24.1 & $<.0001$ & 201 & 54.0 & 208 & 55.9 & .6060 \\
\hline Tricuspid valve & 162 & 28.7 & 139 & 5.7 & $<.0001$ & 66 & 17.7 & 63 & 16.9 & .7714 \\
\hline Valve + CABG & 141 & 25.0 & 423 & 17.3 & $<.0001$ & 93 & 25.0 & 107 & 28.8 & .2470 \\
\hline Previous medications & & & & & & & & & & \\
\hline ACE & 201 & 35.7 & 930 & 38.1 & .2992 & 131 & 35.2 & 133 & 35.8 & .8782 \\
\hline ADP & 9 & 1.6 & 104 & 4.3 & .0027 & 8 & 2.2 & 11 & 3.0 & .4857 \\
\hline
\end{tabular}


TABLE 2. Continued

\begin{tabular}{|c|c|c|c|c|c|c|c|c|c|c|}
\hline \multirow[b]{3}{*}{ Variables } & \multicolumn{5}{|c|}{ Total sample $(\mathrm{N}=\mathbf{3 0 1 4})$} & \multicolumn{5}{|c|}{ Propensity-matched sample $(\mathrm{N}=744)$} \\
\hline & \multicolumn{2}{|c|}{ AF ablated $(N=565)$} & \multicolumn{2}{|c|}{ No $A F(N=2449)$} & \multirow[b]{2}{*}{$P$ value } & \multicolumn{2}{|c|}{ AF ablated $(\mathrm{N}=372)$} & \multicolumn{2}{|c|}{ No AF $(N=372)$} & \multirow[b]{2}{*}{$P$ value } \\
\hline & $\mathbf{n}$ & $\%$ & $\mathbf{n}$ & $\%$ & & $\mathbf{n}$ & $\%$ & $\mathbf{n}$ & $\%$ & \\
\hline Aspirin & 121 & 21.4 & 977 & 40.0 & $<.0001$ & 89 & 23.9 & 100 & 26.9 & .3542 \\
\hline Beta-blockers & 362 & 64.1 & 1358 & 55.6 & .0002 & 220 & 59.1 & 227 & 61.0 & .6003 \\
\hline Warfarin & 88 & 15.6 & 315 & 12.9 & .0877 & 42 & 11.3 & 39 & 10.5 & .7240 \\
\hline Nonstatin & 28 & 5.0 & 120 & 4.9 & .9559 & 15 & 4.0 & 23 & 6.2 & .1828 \\
\hline Statin & 240 & 42.5 & 1299 & 53.0 & $<.0001$ & 162 & 43.6 & 175 & 47.0 & .3383 \\
\hline \multicolumn{11}{|l|}{ Discharge medications } \\
\hline ACE & 129 & 23.7 & 608 & 25.9 & .2899 & 93 & 25.0 & 97 & 26.1 & .7367 \\
\hline ADP & 17 & 3.1 & 509 & 21.3 & $<.0001$ & 16 & 4.3 & 22 & 5.9 & .3177 \\
\hline Aspirin & 500 & 90.1 & 2187 & 91.8 & .2009 & 332 & 89.3 & 341 & 91.7 & .2615 \\
\hline Beta-blockers & 381 & 70.0 & 2063 & 87.0 & $<.0001$ & 284 & 76.3 & 277 & 74.5 & .5512 \\
\hline Warfarin & 472 & 85.2 & 687 & 28.8 & $<.0001$ & 293 & 78.8 & 300 & 80.7 & .5234 \\
\hline Nonstatin & 23 & 4.1 & 99 & 4.0 & .9754 & 15 & 4.0 & 23 & 6.2 & .1828 \\
\hline Statin & 406 & 71.9 & 1854 & 75.7 & .0571 & 271 & 72.9 & 282 & 75.8 & .3559 \\
\hline Ambler score $($ mean $\pm \mathrm{SD})$ & \multicolumn{2}{|c|}{$8.4 \pm 8.2$} & \multicolumn{2}{|c|}{$4.8 \pm 6.5$} & $<.0001$ & \multicolumn{2}{|c|}{$6.3 \pm 6.1$} & \multicolumn{2}{|c|}{$6.9 \pm 8.0$} & .2218 \\
\hline
\end{tabular}

$A F$, Atrial fibrillation; $S D$, standard deviation; $A A$, antiarrhythmic; $C H A D S_{2}$, Congestive heart failure, Hypertension, Age $>75$ years, Diabetes, previous Stroke; $B M I$, body mass index; $C H F$, congestive heart failure; $N Y H A$, New York Heart Association; $C O P D$, chronic obstructive pulmonary disease; $P V D$, peripheral vascular disease; $H T N$, hypertension; $M I$, myocardial infarction; $T I A$, transient ischemic attack; $C A D$, coronary artery disease; $C A B G$, coronary artery bypass grafting; $A C E$, angiotensin-converting enzyme; $A D P$, adenosine diphosphate.

none of these trials were powered or followed to look at whether sinus restoration improved survival or reduced other morbidity.

Few studies have attempted to establish an improved survival after the surgical treatment of AF. In a series of 103 patients with persistent AF, Louagie and colleagues ${ }^{30}$ demonstrated an improved survival at 5 years in 37 patients who underwent a Maze procedure compared with controls. They postulated that sinus restoration $(90 \%$ in Maze group vs $33 \%$ in control) explained the difference. ${ }^{30}$ However, in the non-ablated control group, exclusion of the left atrial appendage was associated with lower mortality, suggesting that a reduction in late stroke may play a role in both populations. In a large series of 521 combined mitral and maze cases, Itoh and colleagues ${ }^{31}$ demonstrated that successful

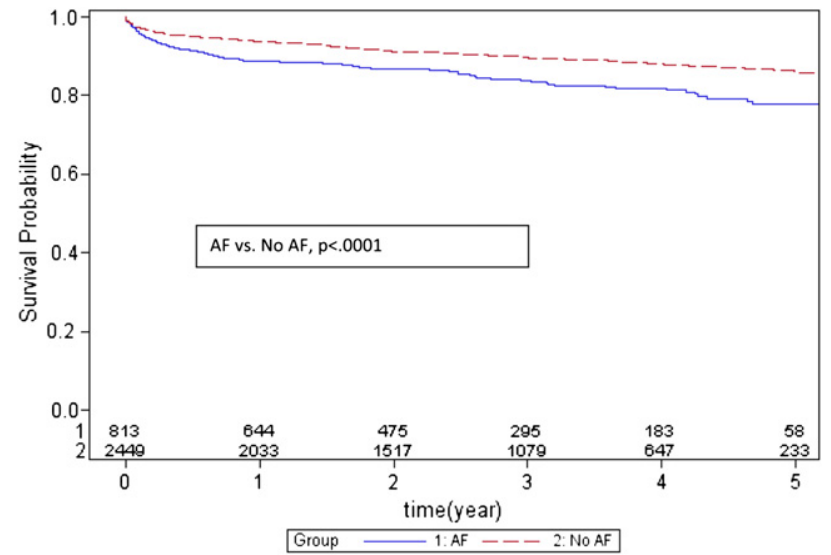

FIGURE 1. Patient survival: history of AF vs no AF. Kaplan-Meier analyses of survival in $\mathrm{AF}(\mathrm{N}=813)$ and no $\mathrm{AF}(\mathrm{N}=2449)$ groups. AF, Atrial fibrillation. sinus restoration improved survival and freedom from stroke. However, these studies preceded current follow-up guidelines and definitions of success. In addition, the use of AA therapy was not considered a failure.

Our propensity-matched analysis demonstrates that in the matched treated population, survival was the same as for patients without a history of AF. Although this could be due to selection bias, it is important to note that those with successful sinus restoration did have improved survival compared with those who failed treatment. The elimination of the left atrial appendage also may be a factor. The role of both these factors on late stroke and survival merits future investigation.

In North America, only $38 \%$ of patients with a history of $\mathrm{AF}$ receive an $\mathrm{AF}$ correction procedure. ${ }^{10,32}$ For some time,

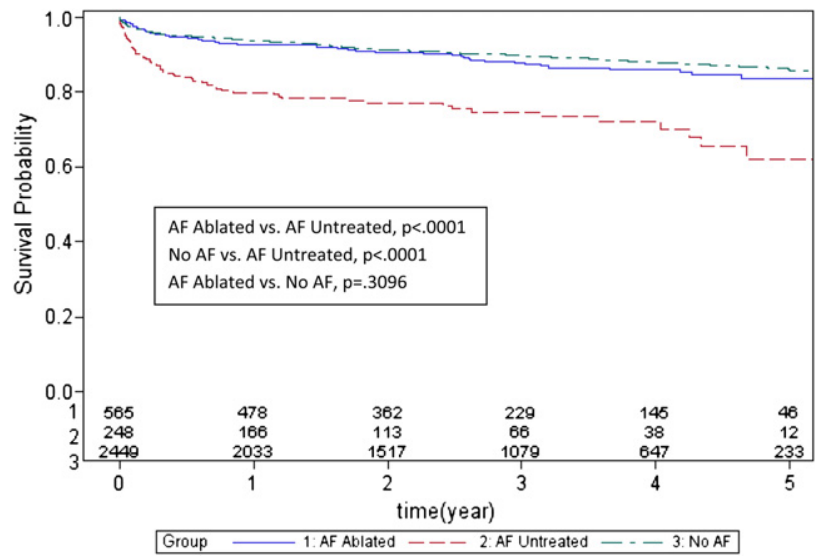

FIGURE 2. Patient survival: no AF vs AF ablated vs AF untreated. Kaplan-Meier analyses of survival for AF ablated $(\mathrm{N}=565)$, AF untreated $(\mathrm{N}=248)$, and no $\mathrm{AF}(\mathrm{N}=2449)$ groups. $A F$, Atrial fibrillation. 


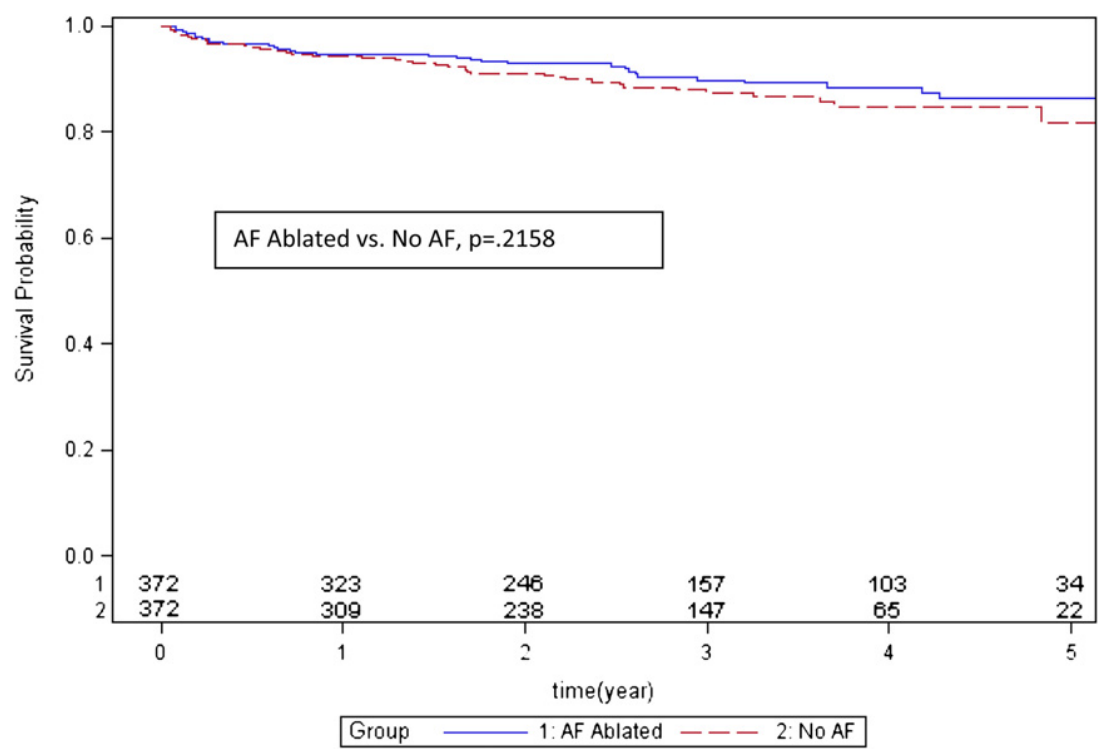

FIGURE 3. Propensity-matched patient survival: AF ablated vs no AF. Kaplan-Meier analyses of survival for propensity-matched AF ablated $(\mathrm{N}=372)$ and no $\mathrm{AF}(\mathrm{N}=372)$ groups. $A F$, Atrial fibrillation.

we have thought that if AF can be eliminated at the time of cardiac surgery, survival should improve to that of patients without a history of $\mathrm{AF}^{5}$ Our study is the first propensity-matched analysis to demonstrate that survival in a population in whom AF was surgically treated in accordance with the Heart Rhythm Society guidelines is comparable to that of patients without AF. Because successful sinus restoration seems to have an impact, an effort should be made to optimize success at the time of surgery. This may have future implications on lesion set selection.

\section{Limitations}

There are several limitations to our study that restrict our ability to definitively state that AF treatment improved survival. The patient sample may not be adequate. Further, although the patients were matched with accepted statistical methodology, there may still be inherent differences in the population that cannot be equated. Despite our best efforts, we may have "selected out" the healthy patients who were destined to have comparable survival. In addition, because of the relatively small sample, we do not know the effect

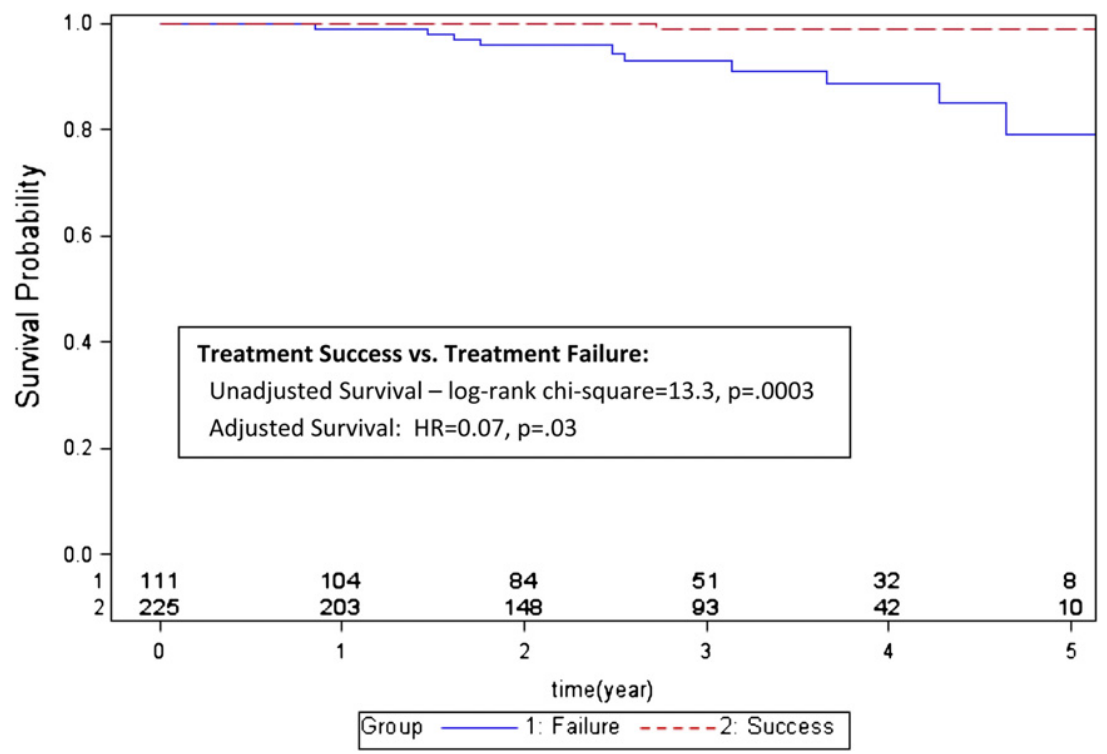

FIGURE 4. Survival in AF ablated group: "success" (freedom from AF and AA therapy at 1 year) vs "failure." Kaplan-Meier analyses of survival for patients in AF ablated group who had no episode of AF lasting more than 30 seconds within the first year, were free of AA medications (success, $\mathrm{N}=225$ ), and had $\mathrm{AF}$ within 1 year or were taking AA medications (failure, $\mathrm{N}=111$ ). $H R$, Hazard ratio. 
TABLE 3. Comparision of characteristics of successful versus unsuccessful AF treatment patients: Total sample and propensity-matched sample 1 year after treatment

\begin{tabular}{|c|c|c|c|c|c|c|c|c|c|c|}
\hline \multirow[b]{3}{*}{ Variables } & \multicolumn{5}{|c|}{ Total sample } & \multicolumn{5}{|c|}{ Propensity-matched sample } \\
\hline & \multicolumn{2}{|c|}{ Success $(\mathbf{N}=\mathbf{2 0 3})$} & \multicolumn{2}{|c|}{ Failure $(N=104)$} & \multirow[b]{2}{*}{$P$ value } & \multicolumn{2}{|c|}{ Success $(\mathbf{N}=77)$} & \multicolumn{2}{|c|}{ Failure $(\mathbf{N}=77)$} & \multirow[b]{2}{*}{$P$ value } \\
\hline & $\mathbf{n}$ & $\%$ & $\mathbf{n}$ & $\%$ & & $\mathbf{n}$ & $\%$ & $\mathbf{n}$ & $\%$ & \\
\hline \multicolumn{11}{|l|}{ Demographics } \\
\hline Age (mean $\pm \mathrm{SD})$ & \multicolumn{2}{|c|}{$64.4 \pm 11.8$} & \multicolumn{2}{|c|}{$68.9 \pm 11.8$} & .0017 & \multicolumn{2}{|c|}{$67.9 \pm 10.9$} & \multicolumn{2}{|c|}{$67.6 \pm 12.0$} & .8498 \\
\hline Male & 118 & 58.1 & 53 & 51.0 & .2315 & 42 & 54.5 & 44 & 57.1 & .7455 \\
\hline Race & & & & & .6895 & & & & & 1.0000 \\
\hline White & 157 & 77.3 & 87 & 83.7 & & 62 & 80.5 & 62 & 80.5 & \\
\hline AA & 10 & 4.9 & 4 & 3.8 & & 3 & 3.9 & 3 & 3.9 & \\
\hline Hispanic & 3 & 1.5 & 2 & 1.9 & & 2 & 1.3 & 2 & 1.3 & \\
\hline Asian & 2 & 1.0 & 1 & 1.0 & & 1 & 2.2 & 1 & 2.2 & \\
\hline Other & 31 & 15.3 & 10 & 9.6 & & 9 & 11.7 & 9 & 11.7 & \\
\hline \multicolumn{11}{|l|}{ Health characteristics } \\
\hline $\mathrm{CHADS}_{2}($ mean $\pm \mathrm{SD})$ & \multicolumn{2}{|c|}{$1.4 \pm 1.2$} & & & .0115 & & 1.2 & & 1.1 & .8869 \\
\hline $\mathrm{BMI}($ mean $\pm \mathrm{SD})$ & & 6.3 & & 5.7 & .4909 & & 4.0 & & 5.6 & .5627 \\
\hline Smoker & 61 & 30.0 & 30 & 28.8 & .8271 & 27 & 35.1 & 24 & 31.2 & .6075 \\
\hline Diabetes & 24 & 11.8 & 17 & 16.3 & .2701 & 9 & 11.7 & 10 & 13.0 & .8064 \\
\hline Hypertension & 114 & 56.2 & 56 & 53.8 & .6998 & 43 & 55.8 & 40 & 51.9 & .6277 \\
\hline $\mathrm{CHF}$ & 60 & 29.6 & 42 & 40.4 & .0566 & 24 & 31.2 & 27 & 35.1 & .6075 \\
\hline NYHA & & & & & .3968 & & & & & .9619 \\
\hline Class 1 & 39 & 19.2 & 18 & 17.3 & & 12 & 15.6 & 14 & 18.2 & \\
\hline Class 2 & 105 & 43.5 & 48 & 37.9 & & 41 & 53.3 & 38 & 49.3 & \\
\hline Class 3 & 55 & 30.6 & 33 & 26.1 & & 22 & 28.6 & 23 & 29.9 & \\
\hline Class 4 & 4 & 4.4 & 5 & 8.4 & & 2 & 2.6 & 2 & 2.6 & \\
\hline Renal failure & 3 & 1.5 & 5 & 4.8 & .0830 & 2 & 2.6 & 2 & 2.6 & 1.0000 \\
\hline Previous dialysis & 0 & 0.0 & 1 & 1.0 & .1617 & 0 & 0.0 & 0 & 0.0 & 1.0000 \\
\hline COPD & 21 & 10.3 & 12 & 11.5 & .7493 & 8 & 10.4 & 8 & 10.4 & 1.0000 \\
\hline PVD & 10 & 4.9 & 4 & 3.8 & .6677 & 2 & 2.6 & 2 & 2.6 & 1.0000 \\
\hline Moderate pulmonary HTN & 71 & 35.0 & 37 & 35.6 & .9168 & 28 & 36.4 & 26 & 33.8 & .7356 \\
\hline Severe pulmonary HTN & 46 & 22.7 & 27 & 26.0 & .5202 & 18 & 23.4 & 17 & 22.1 & .8475 \\
\hline MI & 12 & 5.9 & 11 & 10.6 & .1417 & 4 & 5.2 & 6 & 7.8 & .5131 \\
\hline Angina & 19 & 9.4 & 11 & 10.6 & .7339 & 7 & 9.1 & 9 & 11.7 & .5974 \\
\hline Stroke/TIA & 16 & 7.9 & 11 & 10.6 & .4300 & 9 & 11.7 & 7 & 9.1 & .5974 \\
\hline Family history CAD & 38 & 18.7 & 20 & 19.2 & .9137 & 13 & 16.9 & 14 & 18.2 & .8322 \\
\hline Surgical characteristics & & & & & & & & & & \\
\hline No. of diseased vessels (mean \pm SD) & & & & & .2829 & & 1.1 & & 1.1 & .9018 \\
\hline Left main disease & 8 & 4.0 & 3 & 2.9 & .6426 & 1 & 1.3 & 3 & 3.9 & .3109 \\
\hline Preoperative creatinine (mean \pm SD) & & & & & .0244 & & 0.4 & & 0.6 & .9887 \\
\hline Surgical incidence & & & & & .0054 & & & & & .5594 \\
\hline $1 \mathrm{st}$ & 175 & 86.2 & 77 & 74.0 & & 64 & 83.1 & 63 & 81.8 & \\
\hline 2nd & 27 & 13.3 & 22 & 21.2 & & 12 & 15.6 & 14 & 18.2 & \\
\hline $3+$ & 1 & 0.5 & 5 & 4.8 & & 1 & 1.3 & 0 & 0.0 & \\
\hline Indication of surgery & & & & & .2816 & & & & & .9999 \\
\hline Elective & 189 & 93.1 & 100 & 96.2 & & 75 & 97.0 & 74 & 96.1 & \\
\hline Emergency & 0 & 0.0 & 0 & 0.0 & & 0 & 0.0 & 0 & 0.0 & \\
\hline Urgent & 14 & 6.9 & 4 & 3.8 & & 2 & 2.6 & 3 & 3.9 & \\
\hline Type of surgery & & & & & & & & & & \\
\hline CABG & 53 & 26.1 & 32 & 30.8 & .3877 & 29 & 37.7 & 23 & 29.9 & .3066 \\
\hline Aortic valve & 52 & 25.6 & 26 & 25.0 & .9066 & 21 & 27.3 & 18 & 23.4 & .5783 \\
\hline Mitral valve & 123 & 60.6 & 76 & 73.1 & .0301 & 53 & 68.8 & 53 & 68.8 & 1.0000 \\
\hline Tricuspid valve & 54 & 26.6 & 41 & 39.4 & .0214 & 27 & 35.1 & 26 & 33.8 & .8653 \\
\hline Valve + CABG & 44 & 21.7 & 27 & 26.0 & .3992 & 26 & 33.8 & 19 & 24.7 & .2149 \\
\hline Previous medications & & & & & & & & & & \\
\hline ACE & 71 & 35.1 & 42 & 40.8 & .3358 & 34 & 44.2 & 33 & 42.9 & .8709 \\
\hline $\mathrm{ADP}$ & 1 & 0.5 & 2 & 1.9 & .2241 & 1 & 1.3 & 0 & 0.0 & .3157 \\
\hline
\end{tabular}




\begin{tabular}{|c|c|c|c|c|c|c|c|c|c|c|}
\hline \multirow[b]{3}{*}{ Variables } & \multicolumn{5}{|c|}{ Total sample } & \multicolumn{5}{|c|}{ Propensity-matched sample } \\
\hline & \multicolumn{2}{|c|}{ Success $(N=203)$} & \multicolumn{2}{|c|}{ Failure $(N=104)$} & \multirow[b]{2}{*}{$P$ value } & \multicolumn{2}{|c|}{ Success $(N=77)$} & \multicolumn{2}{|c|}{ Failure $(\mathbf{N}=77)$} & \multirow[b]{2}{*}{$P$ value } \\
\hline & $\mathbf{n}$ & $\%$ & $\mathbf{n}$ & $\%$ & & $\mathbf{n}$ & $\%$ & $\mathbf{n}$ & $\%$ & \\
\hline Aspirin & 39 & 19.2 & 17 & 16.3 & .5383 & 13 & 16.9 & 11 & 14.3 & .6568 \\
\hline Beta-blockers & 130 & 64.0 & 59 & 56.7 & .2128 & 42 & 54.5 & 43 & 55.8 & .8713 \\
\hline Warfarin & 24 & 11.8 & 12 & 11.5 & .9416 & 7 & 9.1 & 8 & 10.4 & .7858 \\
\hline Nonstatin & 12 & 5.9 & 6 & 5.8 & .9600 & 4 & 5.2 & 4 & 5.2 & 1.0000 \\
\hline Statin & 78 & 38.4 & 45 & 43.3 & .4122 & 31 & 40.3 & 33 & 42.9 & .7437 \\
\hline \multicolumn{11}{|l|}{ Discharge medications } \\
\hline $\mathrm{ACE}$ & 49 & 24.4 & 26 & 25.2 & .8685 & 24 & 31.2 & 19 & 24.7 & .3691 \\
\hline $\mathrm{ADP}$ & 5 & 2.5 & 0 & 0.0 & .1074 & 0 & 0.0 & 0 & 0.0 & 1.0000 \\
\hline Aspirin & 185 & 91.1 & 99 & 95.2 & .2010 & 66 & 85.7 & 72 & 93.5 & .5131 \\
\hline Beta-blockers & 133 & 66.8 & 70 & 69.3 & .6652 & 51 & 66.2 & 52 & 67.5 & .8641 \\
\hline Warfarin & 176 & 87.1 & 91 & 87.5 & .9265 & 67 & 87.0 & 69 & 89.6 & .6159 \\
\hline Nonstatin & 12 & 5.9 & 3 & 2.9 & .2443 & 1 & 1.3 & 2 & 2.6 & .5599 \\
\hline Statin & 147 & 72.4 & 76 & 73.1 & .9018 & 51 & 66.2 & 56 & 72.7 & .3816 \\
\hline Ambler score (mean $\pm \mathrm{SD})$ & \multicolumn{2}{|c|}{$7.0 \pm 7.3$} & \multicolumn{2}{|c|}{$10.3 \pm 8.3$} & .0004 & \multicolumn{2}{|c|}{$7.8 \pm 8.7$} & \multicolumn{2}{|c|}{$7.9 \pm 6.3$} & .8819 \\
\hline
\end{tabular}

of nontreatment in this group; however, we do know that patients had a worse survival when treatment failed. AF ablation was not performed on these patients when the surgeon judged the procedure too complex or the patients were too ill and the risk outweighed the potential benefit; generally, these patients were likely to have worse survival. Thus, little information can be gained from the untreated population in this study. Because the untreated patients were different from the treated patients, we did not find an adequate sample for matching this group. Other limitations include a lack of knowledge of late causes of death that may be unrelated to AF. Late adverse events were not investigated; only midterm survival was investigated. Longer follow-up will provide a more definitive answer.

\section{CONCLUSIONS}

In matched patients with a history of AF who underwent ablation during concomitant surgery, there was no difference in early or midterm survival compared with patients without a preoperative history of AF. The $67 \%$ of patients who were free of AA therapy and AF at 1 year had better long-term survival, and $78 \%$ of patients in sinus rhythm

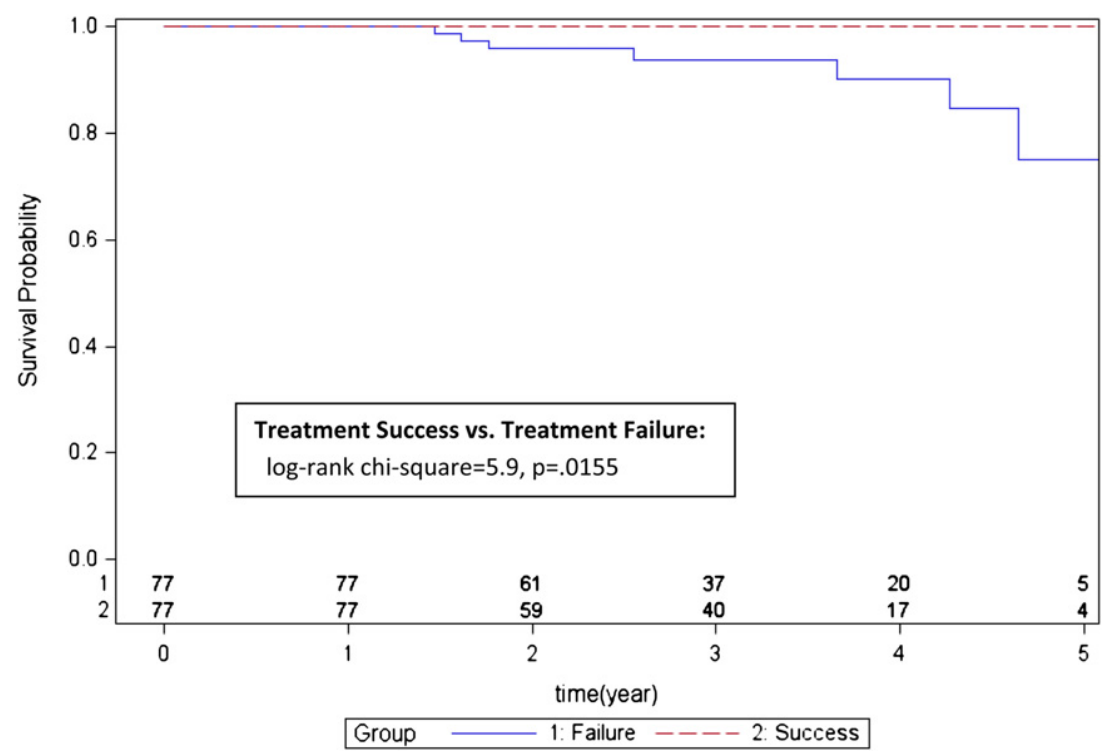

FIGURE 5. Survival after 1 year in propensity-matched patients: "success" (freedom from AF and AA therapy at 1 year) vs failure. Kaplan-Meier analysis of survival after 1 year in propensity-matched patients; a comparison of AF treatment success $(\mathrm{N}=77)$ and failure $(\mathrm{N}=77)$ groups. 
at last follow-up showed a trend $(P=.06)$ toward improved survival compared with patients in AF. In matched patients, successful treatment predicted survival, even after 1 year. Because several prior studies have indicated reduced survival in patients with a history of AF, these results are encouraging and merit further investigation. This may have implications for a broader application of AF surgery in low-risk patients.

\section{References}

1. Kannel WB, Abbott RD, Savage DD, McNamara PM. Epidemiologic features of chronic atrial fibrillation: the Framingham study. N Engl J Med. 1982;306: 1018-22.

2. Stewart S, Hart CL, Hole DJ, McMurray JJ. A population-based study of the long-term risks associated with atrial fibrillation: 20-year follow-up of the Renfrew/Paisley study. Am J Med. 2002;113:359-64.

3. Wyse DG, Waldo AL, DiMarco JP, Domanski MJ, Rosenberg Y, Schron EB, et al. A comparison of rate control and rhythm control in patients with atrial fibrillation. N Engl J Med. 2002;347:1825-33.

4. Corley SD, Epstein AE, DiMarco JP, Domanski MJ, Geller N, Greene HL, et al. Relationships between sinus rhythm, treatment, and survival in the Atrial Fibrillation Follow-Up Investigation of Rhythm Management (AFFIRM) Study. Circulation. 2004;109:1509-13.

5. Quader MA, McCarthy PM, Gillinov AM, Alster JM, Cosgrove DM 3rd, Lytle BW, et al. Does preoperative atrial fibrillation reduce survival after coronary artery bypass grafting? Ann Thorac Surg. 2004;77:1514-24.

6. Ngaage DL, Schaff HV, Mullany CJ, Sundt TM 3rd, Dearani JA, Barnes S, et al. Does preoperative atrial fibrillation influence early and late outcomes of coronary artery bypass grafting? J Thorac Cardiovasc Surg. 2007;133:182-9.

7. Ngaage DL, Schaff HV, Barnes SA, Sundt TM 3rd, Mullany CJ, Dearani JA, et al. Prognostic implications of preoperative atrial fibrillation in patients undergoing aortic valve replacement: is there an argument for concomitant arrhythmia surgery? Ann Thorac Surg. 2006;82:1392-9.

8. Schulenberg R, Antonitsis P, Stroebel A, Westaby S. Chronic atrial fibrillation is associated with reduced survival after aortic and double valve replacement. Ann Thorac Surg. 2010;89:738-44.

9. Ngaage DL, Schaff HV, Mullany CJ, Barnes S, Dearani JA, Daly RC, et al. Influence of preoperative atrial fibrillation on late results of mitral repair: is concomitant ablation justified? Ann Thorac Surg. 2007;84:434-43.

10. Gammie JS, Haddad M, Milford-Beland S, Welke KF, Ferguson TB Jr, O'Brien SM, et al. Atrial fibrillation correction surgery: lessons from the Society of Thoracic Surgeons National Cardiac Database. Ann Thorac Surg. 2008;85: 909-14.

11. Stulak JM, Sundt TM 3rd, Dearani JA, Daly RC, Orsulak TA, Schaff HV. Tenyear experience with the Cox-maze procedure for atrial fibrillation: how do we define success? Ann Thorac Surg. 2007;83:1319-24.

12. Damiano RJ Jr, Gaynor SL, Bailey M, Prasad S, Cox JL, Boineau JP, et al. The long-term outcome of patients with coronary disease and atrial fibrillation undergoing the Cox maze procedure. J Thorac Cardiovasc Surg. 2003;126:2016-21.

13. McCarthy PM, Kruse J, Shalli S, Ilkhanoff L, Goldberger JJ, Kadish AH, et al. Where does atrial fibrillation surgery fail? Implications for increasing effectiveness of ablation. J Thorac Cardiovasc Surg. 2010;139:860-7.

14. Lee R, Kruse J, McCarthy PM. Surgery for atrial fibrillation. Nat Rev Cardiol. 2009;6:505-13.

15. Cox JL, Ad N, Palazzo T. Impact of the maze procedure on the stroke rate in patients with atrial fibrillation. J Thorac Cardiovasc Surg. 1999;118:833-40.

16. Calkins H, Brugada J, Packer DL, Cappato R, Chen SA, Crijns HJ, et al. HRS/ EHRA/ECAS Expert Consensus Statement on Catheter and Surgical Ablation of Atrial Fibrillation: Recommendations for Personnel, Policy, Procedures and Follow-Up. A report of the Heart Rhythm Society (HRS) Task Force on Catheter and Surgical Ablation of Atrial Fibrillation. Heart Rhythm. 2007;4: 816-61.

17. Austin P. Some methods of propensity-score matching had superior performance to others: results of an empirical investigation and Monte Carlo simulations. Biom J. 2009;51:171-84.

18. Sergeant P, Lesaffre E, Flameng W, Suy R, Blackstone E. The return of clinically evident ischemia after coronary artery bypass grafting. Eur J Cardiothorac Surg. 1991;5:447-57.
19. Doty DB. Surgical treatment of atrial fibrillation. Heart Lung Circ. 2004;13: 280-7.

20. Gillinov AM, Bhavani S, Blackstone EH, Rajeswaran J, Svensson LG, Navia JL, et al. Surgery for permanent atrial fibrillation: impact of patient factors and lesion set. Ann Thorac Surg. 2006;82:502-14.

21. Nitta T. Surgery for atrial fibrillation: a worldwide review. Semin Thorac Cardiovasc Surg. 2007;19:3-8.

22. Nitta T, Ishii Y, Ogasawara H, Sakamoto S, Miyagi Y, Yamada K, et al. Initial experience with the radial incision approach for atrial fibrillation. Ann Thorac Surg. 1999;68:805-11.

23. Doty JR, Doty DB, Jones KW, Flores JH, Mensah M, Reid BB, et al. Comparison of standard Maze III and radiofrequency Maze operations for treatment of atrial fibrillation. J Thorac Cardiovasc Surg. 2007;133:1037-44.

24. Srivastava V, Kumar S, Javali S, Rajesh TR, Pai V, Khandekar J, et al. Efficacy of three different ablative procedures to treat atrial fibrillation in patients with valvular heart disease: a randomised trial. Heart Lung Circ. 2008;17:232-40.

25. Jessurun ER, van Hemel NM, Defauw JJ, Brutel De La Riviere A, Stofmeel MA, Kelder JC, et al. A randomized study of combining maze surgery for atrial fibrillation with mitral valve surgery. J Cardiovasc Surg (Torino). 2003;44:9-18.

26. Khargi K, Deneke T, Haardt H, Lemke B, Grewe P, Muller KM, et al. Saline-irrigated, cooled-tip radiofrequency ablation is an effective technique to perform the maze procedure. Ann Thorac Surg. 2001;72:S1090-5.

27. Akpinar B, Guden M, Sagbas E, Sanisoglu I, Ozbek U, Caynak B, et al. Combined radiofrequency modified maze and mitral valve procedure through a port access approach: early and mid-term results. Eur J Cardiothorac Surg. 2003; 24:223-30.

28. de Lima GG, Kalil RA, Leiria TL, Hatem DM, Kruse CL, Abrahao R, et al. Randomized study of surgery for patients with permanent atrial fibrillation as a result of mitral valve disease. Ann Thorac Surg. 2004;77:2089-95.

29. Abreu Filho CA, Lisboa LA, Dallan LA, Spina GS, Grinberg M, Scanavacca M, et al. Effectiveness of the maze procedure using cooled-tip radiofrequency ablation in patients with permanent atrial fibrillation and rheumatic mitral valve disease. Circulation. 2005;112(9 Suppl):I20-5.

30. Louagie Y, Buche M, Eucher P, Schoevaerdts JC, Gerard M, Jamart J, et al. Improved patient survival with concomitant Cox Maze III procedure compared with heart surgery alone. Ann Thorac Surg. 2009;87:440-6.

31. Itoh A, Kobayashi J, Bando K, Niwaya K, Tagusari O, Nakajima H, et al. The impact of mitral valve surgery combined with maze procedure. Eur J Cardiothorac Surg. 2006;29:1030-5.

32. Cox JL. The longstanding, persistent confusion surrounding surgery for atrial fibrillation. J Thorac Cardiovasc Surg. 2010;139:1374-86.

\section{Discussion}

Dr John Doty (Murray, Utah). This study emphasizes the importance of concomitant treatment of AF, particularly your last graphs. Your follow-up is relatively short, but we may see that those patients have improved survival by treatment of AF. There is ongoing debate about the wide range of operations that are used for the treatment of AF. Would you describe the particular procedures your group uses for treatment of $\mathrm{AF}$ and how you decide which patient gets what procedure in this complex group?

Dr Lee. I have another article that I am putting together that looks at $\mathrm{AF}$ by lesion set, and in general our results are approximately $75 \%$ to $90 \%$ depending on the type of procedure that we use in the patient population. Our rule of thumb is essentially this. The American College of Cardiology/American Heart Association recommends treating everybody with $\mathrm{AF}$ if it does not add anything more to the procedure. So if you are performing mitral valve surgery, you are fairly obligated to do a full left-sided Maze and strongly consider a right side, especially as more data emerge that doing both by atrial lesion sets may improve AF outcomes. On the flip side, if you have an elderly person undergoing CABG, especially off-pump, and you are not going to open the heart, well, it may be reasonable to do pulmonary vein isolation 
alone. We segment it by procedure and by how AF affects the patient. If $\mathrm{AF}$ is a real problem, we make every effort to treat it with the biatrial lesion set. If it is paroxysmal, especially if we are not going to open the heart anyway, we would do a more limited lesion set like a PVI alone. And for those patients in between those extremes, we perhaps do a left-sided only Maze.

Dr Doty. Maybe you could clarify a bit more. When you say lesion sets, what energy modality are you using? You did mention in your article sometimes you have cut and sew as an option.

Dr Lee. In the article you wrote 2 years ago, you showed that cut and sew is probably better than everything else, and even though some of the other reports conflict with that, we have a similar experience. Now if you have a patient with $\mathrm{AF}$ and that is the number 1 problem, you are going to do a cut-and-sew Maze. If you have a patient who has paroxysmal or perhaps long-standing persistent and not permanent AF, you will perform bipolar RF. The majority of our patients are older, more frail, and so approximately $75 \%$ of our patients receive bipolar radiofrequency with supplementation at the annulus with cryothermia.

Dr Doty. We all recognize that some surgeons may not be willing to add an additional procedure for AF to what they are doing, particularly if it is complex. Can you estimate for us how much additional time it takes your group to do some type of ablative or incisional Maze procedure?

Dr Lee. That is important, but even the experience of our group is not as important as the experience of the whole population. Gammie and colleagues wrote an article using the Society of Thoracic Surgeons' database. In that added meta-analysis, they showed that it added 9 minutes to crossclamp time and 9 additional minutes to bypass time in general. With the bypass time, you are clamping the beating heart anyway. I think it is even less than that in many instances, so if we are using bipolar radiofrequency we go on pump. We do the pulmonary vein isolations on pump. We stop the heart. We do the connecting lesions, and that really does not take more than 1 minute of clamp time. Then we unclamp and do the right-sided lesions if we are going to do it, and again even if you do multiple lesions it is 40 seconds per burn times 3 . It is just a couple minutes per lesion, so in all it adds approximately 18 minutes at the most, but in general, more like 10 minutes for us.

Dr Doty. Your group is similar to ours. We have a strong bias to treat everyone who presents with AF with some type of procedure during their concomitant operation. I am particularly interested in the subset of patients who had existing AF but did not receive a concomitant procedure. You noted these patients were more ill and had more comorbidities. If that is the case, shouldn't these patients be the very ones who receive a concomitant operation, and, if not, can you explain which patients in whom you would not perform an AF operation?
Dr Lee. I did not analyze those data, but you know we have shifted. Now we look at how many patients we have with AF, and now we are at $90 \%$ of patients treated for AF, so we have had a paradigm shift. We have a group we assembled right around the beginning of that time period, and as we have gone on we have increased the application, but we also use alternate energy sources, so if you have a redo, for example, say a redo mitral that is pretty stuck, you don 't want to dissect out the whole heart. I don't want to do a cut-and-sew Maze in that patient. I don't even want to do bipolar radiofrequency in that patient because I don't want to get around the left-sided veins. But what we will do is use a CryoCath (Medtronic Corporation, Minneapolis, Minn) around the entire surface or Frigitronics cryo (Atricure Corporation, Cincinnati, Ohio), 1 of those 2, and I would say we are down to not operating on approximately $10 \%$ of the patients. In general, if we are not going to open the heart, say it is an 85-year-old patient with a redo aortic valve, we are probably just going to do the redo aortic valve. If it is a CABG, especially a redo, we probably would let that go if it is an older or frail patient. That being said, now we are down to approximately $10 \%$ or less of patients with a history of AF who are in that category.

Dr Doty. Let me clarify. Are there specific patients in whom you would not perform a concomitant operation? You mentioned redos, if they are older. Are there other criteria you generally use?

Dr Lee. If the patient is older, we are not going to open the heart. Even if the patient is old and a redo and we are going to perform mitral valve surgery, that is still easy to do a left sided, but the guidelines state that it adds to the operation significantly, so I would not open the heart in a 90-year-old patient with redo aortic valves and patent grafts. That is clearly a patient I would not operate on. I cannot give you an individual patient because it is a caseby-case decision, but that is clearly to the extreme where I say no.

Dr William Northrup (Atlanta, Ga). I wonder if the phrase "expected survival" is a little misleading. For instance, when we say that mitral valve repair has the potential to restore expected survival, we are comparing it with a normal matched population. In this case, you are comparing 2 surgical subgroups. When I first read the article, I thought this is pretty exciting. Nevertheless, the article presents exciting data.

Dr Lee. Thank you for your comment, and we will take that into consideration for the final article. We did not compare with the general population because we do not know what that survival should be compared with regular cardiac surgery, but we do know what survival should be for similar patients undergoing aortic valve surgery, mitral valve surgery, and CABG, so by the word "expected," that is what we meant, and we did restore it to that population. 